\title{
EDITORIAL
}

\section{Dank und neuer Auftakt: Wechsel in der Schriftleitung}

,Editorials' haben in dieser Zeitschrift Seltenheitswert; es muss dafür schon einen besonderen Anlass geben. Dies war so, als in Heft 2 des Jahrgangs $1985^{1}$ die Redaktion eine gemeinsame europapolitische Stellungnahme vorlegte. Damals kündigten sich folgenreiche integrationspolitische Weichenstellungen an (sie führten schließlich zur „Einheitlichen Europäischen Akte“). Die Zeitschrift habe sich „,keiner Ideologie und keiner wissenschaftlichen Doktrin verschrieben“; dennoch plädierte die Redaktion für die Sicherstellung von „unverzichtbaren Elementen der Europäischen Union“ im Zug der bevorstehenden Reform. Betont wurden erstens die Erfordernisse der demokratischen Legitimation und der Effizienz der Entscheidungsverfahren (wobei Mitentscheidungsbefugnisse des Parlaments und Mehrheitsentscheidungen im Rat gefordert wurden); zweitens eine sinnvolle und eindeutiger definierte Aufgabenteilung zwischen der Union und den Mitgliedstaaten, nach Maßgabe einer Reihe namhaft gemachter Kriterien; sowie drittens das Erfordernis eines Gründungsvertrags für die Union (wie er dann erst im Rahmen des Projekts Maastricht zustande kam). Wenn diese Erfordernisse nicht von allen damaligen zwölf Mitgliedstaaten mitgetragen werden könnten, wäre es besser, die Vertiefung in einem ,kleineren Kreis von integrationswilligen Staaten“ ins Werk zu setzen als ganz auf sie zu verzichten (das war rund ein Jahrzehnt vor der Wiedereröffnung der ,Kerneuropa'-Debatte durch Wolfgang Schäuble und Karl Lamers 1994).

Ein zweites Mal wurde am Ende des 30. Jahrgangs ein Heft der integration mit einem Editorial eröffnet: ${ }^{2}$ nicht so sehr um der Zeitschrift willen, sondern im Blick auf das Fünfzig-Jahre-Jubiläum der Römischen Verträge und angesichts des Mangels eines klaren und konsensfähigen europapolitischen Kurses, wie er vor allem durch das Scheitern des Verfassungsprojekts offenkundig geworden war. Ihm entspreche eine ,,auffällige Mehrdeutigkeit und Unsicherheit der wissenschaftlich angeleiteten Analyse“. Diesmal wurde kein Bekenntnis zu bestimmten Reformzielen abgegeben, sondern nur die Bereitschaft und der Wille bekundet, um weiterführende Analysen und um Beiträge zur sachgerechten Urteilsbildung bemüht zu sein.

Nun aber ist der Anlass für ein Editorial von ganz anderer Art; er hat nicht mit Hoffnungen oder Besorgnissen zur Europapolitik zu tun (dazu finden die Leserinnen und Leser ohnehin Anregungen zum Nachdenken im Heft); die Unterzeichnenden meinen trotzdem, diese Ausgabe der integration sollte mit einem besonderen Wort an die Leser eröffnet werden.

Vor zwanzig Jahren ist die langjährige Schriftleiterin der integration, Dr. Barbara Lippert, in die Redaktion eingetreten. Bald danach, 1993, übernahm sie die Funktion des geschäftsführenden Redaktionsmitglieds, und viele Jahre lang lag die redaktionelle Gesamtverantwortung in ihren Händen. In dieser Zeit hat sie die Zeitschrift zunehmend maßgeblich gestaltet. In ihrer Anfangszeit hatte das Einzelheft gerade mal einen Umfang von gut 40 Sei-

1 Stellungnahme der Redaktion INTEGRATION zur aktuellen Debatte: Die unverzichtbaren Elemente der Europäischen Union, in: integration 2/1985, S. 47-48.

2 Heinrich Schneider: 50 Jahre Römische Verträge - 30 Jahre integration, in: integration 4/2007, S. 367-369. 
ten, das Erscheinungsbild war überaus bescheiden. In der internationalen Fachliteratur zitiert wurden Beiträge eher selten.

Inzwischen ist die integration über die Grenzen des Landes hinaus anerkannt und geschätzt. Autoren sehen die Veröffentlichung eines Aufsatzes als Anerkennung wissenschaftlicher Reputation. Das alles ist nicht einfach das Verdienst von Dr. Barbara Lippert allein. Redaktionsmitglieder, Autoren, aufgeschlossene und kommunikationswillige Leser, Förderer wie das Auswärtige Amt, der Verlag und nicht zuletzt das Institut für Europäische Politik sowie der „Arbeitskreis Europäische Integration“ (die deutsche Sektion der interdisziplinären Fachvereinigung der Europaforscher ECSA) haben dazu dankenswert beigetragen. Aber der Anteil von Dr. Barbara Lippert war doch sozusagen der Löwinnen-Anteil.

Sie hat das Angebot angenommen, künftig als Forschungsdirektorin in der Institutsleitung der „Stiftung Wissenschaft und Politik“ tätig zu sein. Ihre Kolleginnen und Kollegen in der Redaktion und im Institut für Europäische Politik freuen sich über die in diesem Angebot zum Ausdruck kommende Würdigung ihrer Kompetenz in den Bereichen der Forschung, des Wissenschaftsmanagements und der Menschenführung und wünschen ihr aufrichtig alles Gute im neuen Wirkungsbereich.

Dass Dr. Barbara Lippert in ihren bisherigen Funktionen nicht leicht ersetzt werden kann, versteht sich von selbst. Umso erfreulicher ist es, dass die redaktionelle Arbeit auch im Zeichen von Kontinuität weitergeführt werden kann: Die - zusammen mit dem Herausgeber dieses Editorial verantwortende neue Schriftleiterin, Dr. Elfriede Regelsberger, ist schon seit vielen Jahren, so wie das Dr. Barbara Lippert bis jetzt war, Stellvertretende Direktorin des Instituts. Den Lesern der integration und allen mit der Europapolitik politisch oder wissenschaftlich befassten Zeitgenossen sind ihre Fachpublikationen geläufig.

Die Verfasser dieses Editorials möchten an dieser Stelle Dr. Barbara Lippert für die sachlich und menschlich stets überaus erfreuliche Zusammenarbeit danken. Zugleich bitten sie alle Weggefährten der Zeitschrift, insbesondere die bisherigen und die präsumptiven Autoren sowie die Leser, der Redaktion auch künftig ihr Vertrauen zu schenken. Anregungen und kritische Anmerkungen sind und bleiben willkommen, ebenso wie Typoskripte, die nach dem Ablauf des Begutachtungsverfahrens veröffentlicht werden sollen. Wir hoffen, dass die Zeitschrift ihre bisherigen Freunde behält und neue Freunde findet.

Prof. Dr. Heinrich Schneider

Herausgeber
Dr. Elfriede Regelsberger Schriftleitung 